\title{
Bildgebung in der Beckenchirurgie
}

Holger Keil, Paul A. Grützner, Jochen Franke

\section{Einleitung}

Verletzungen des Beckens als dem zentralen Bindeglied zwischen der Wirbelsäule, dem Körperstamm und den unteren Extremitäten sind mit einer Häufigkeit von ca. 5-8\% aller Frakturen insgesamt eine eher seltene Frakturform [1]. Die Häufigkeitsverteilung weist die für viele Frakturen typischen 2 Gipfel auf. Vermehrt treten diese Frakturen bei jungen, tendenziell eher männlichen Patienten im Rahmen von Hochrasanztraumata wie Verkehrsunfällen und Stürzen aus großer Höhe auf, ebenso bei älteren, tendenziell eher weiblichen Patienten im Rahmen von Stürzen aus dem Stand bei reduzierter Knochenqualität und multifaktorieller Sturzneigung. Diese völlig unterschiedlichen Unfallmechanismen und die daraus resultierenden Verletzungen gilt es, für die weitere Therapie zu beachten, insbesondere vor dem Hintergrund der möglichen Begleitverletzungen.

\footnotetext{
Merke

Insbesondere beim jungen Verunfallten nach Begleitverletzungen suchen!
}

Unter dem Begriff der Beckenverletzungen summieren sich Frakturen des Azetabulums mit oder ohne begleitende Instabilität des Hüftgelenkes, knöcherne oder weichteilige Verletzungen des vorderen und hinteren Beckenringes mit Auswirkungen auf die Gesamtstabilität des Beckens. Weiterhin fallen hierunter Frakturen des Sakrums, die entweder im Rahmen von Beckenringverletzungen oder isoliert auftreten können.

Die Beurteilung des Verletzten und der Verletzungen erfordert demnach die Berücksichtigung des Unfallmechanismus und der Begleitverletzungen, da lediglich ca. $20 \%$ der Verletzungen als isolierte Beckenfrakturen auftreten. Dementsprechend ergibt sich die Dringlichkeit und Operationstaktik (schnelle bzw. Notfallstabilisierung vs. semielektive Osteosynthese des stabilisierten Patienten) eines operativen Eingriffs aus der Gesamtverletzungskonstellation und der Begleitsymptomatik.

Zur Thematik der klinischen Untersuchung des Beckens, insbesondere bei Mehrfachverletzten und Polytraumatisierungen, sind in den letzten Jahren Untersuchungen hinsichtlich der Sensitivität und Spezifität der manuellen Stabilitätsprüfung durchgeführt worden. Hier zeigte sich überwiegend eine schlechte Diagnostizierbarkeit instabiler Verletzungen durch die Stabilitätsprüfung bei der gleichzeitigen Befürchtung, dass durch eine forcierte Durchführung eine Blutung verstärkt werden könnte. Die Stabilitätsprüfung wird daher nicht mehr routinemäßig empfohlen [2,3]. \section{Begleitverletzungen weiterhin obligat! \\ Präoperative Bildgebung und Klassifikation}

Eine klinische Untersuchung des vorderen und hinteren Beckenringes ist insbesondere im Hinblick auf

Nach wie vor stellt das klassische Röntgen die initiale Diagnostik bei Verdacht auf Verletzungen des knöchernen Beckens dar. Je nach klinischer Präsentation muss man sich hier bereits Gedanken über die am wahrscheinlichsten verletzte Struktur machen, um die Bildgebung entsprechend vorzusehen.

\section{Beckenring}

Hier ist neben einer Beckenübersicht, die auch das komplette Os ilium mit einschließen sollte, die Durchführung von Inlet- und Outlet-Aufnahmen indiziert. Diese stellen um ca. $45^{\circ}$ nach kaudal und krainal gekippte Projektionen mit Darstellung der Eingangsebene ins kleine Becken (Outlet) bzw. orthograder Darstellung der Foramina obturatoria (Inlet) dar (siehe $>$ Abb. $\mathbf{1} \mathbf{a}-\mathbf{c}$ ).

Damit ist i.d. R. eine suffiziente Beurteilung des vorderen Beckenringes sowie der Gesamtsymmetrie des Beckens möglich. Ein sicherer Frakturausschluss des Sakrums bzw. Stellungsbeurteilung der Sakroiliakalgelenke ist hiermit jedoch nicht möglich, sodass bei entsprechendem Verdacht die Indikation zur CT-Bildgebung großzügig gestellt werden sollte. Erfolgt nach Abwägung keine CTDiagnostik bei Beschwerdeangabe im hinteren Beckenring, sollte zumindest noch eine seitliche Aufnahme des Sakrums erfolgen und engmaschige Röntgenkontrollen nach Mobilisierung durchgeführt werden. 

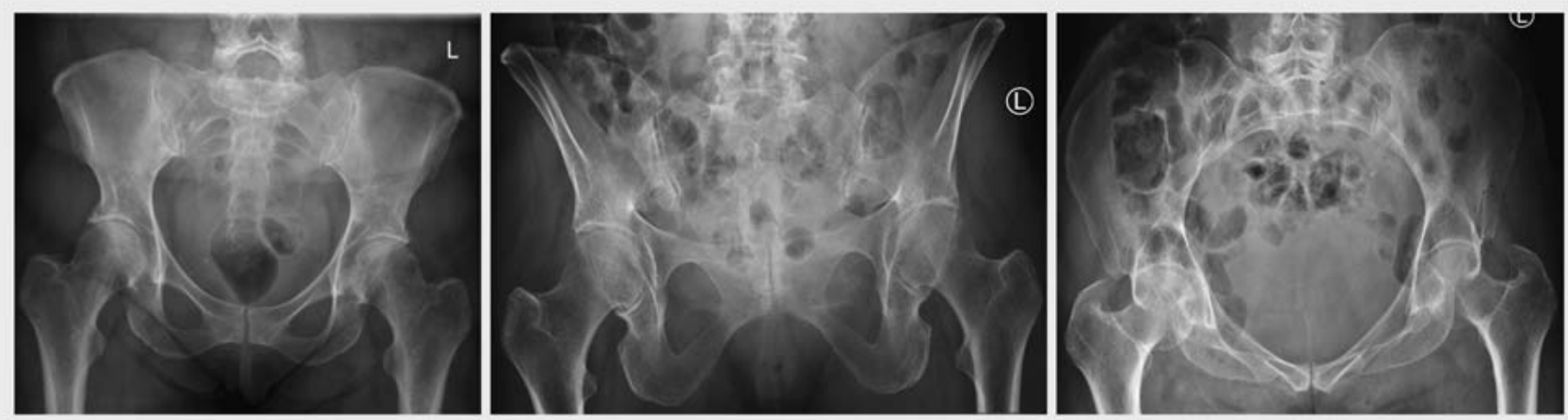

Abb. 1 a A.-p. Projektion, b Inlet-Projektion und c Outlet-Projektion.

\section{Klassifikation}

Beckenringfrakturen werden je nach durch die Verletzung entstandener Instabilität nach AO in Typ A, Typ B oder Typ $C$ eingeteilt. Bei Typ-A-Verletzungen ist die Stabilität des vorderen und hinteren Beckenringes erhalten. Bei Typ-B-Verletzungen besteht eine Instabilität in horizontaler Richtung, dieser Situation entspricht morphologisch eine Stabilitätsunterbrechung von Anteilen des vorderen und eine partielle Instabilität des hinteren Beckenringes zwingend. Bei Typ-C-Verletzungen sind vordere und hintere Stabilisatoren komplett unterbrochen, sodass eine horizontale und vertikale Instabilität entsteht.

Die Subklassifikation ergibt sich aus den betroffenen anatomischen Strukturen und ist in $\mathbf{A} \mathbf{A b b} \mathbf{2}$ dargestellt. Durch diese Klassifikation ist seitengetrennt eine vollständige codierte Erfassung der möglichen Verletzungen zu erreichen.

Eine Operationsindikation besteht je nach resultierender Instabilität. So ist bei Typ-A-Frakturen i. d. R. eine konservative Therapie möglich, während bei Typ-B-Frakturen insbesondere mit Symphysenruptur und Dislokation in Außenrotation - eine operative Stabilisierung indiziert ist. Typ-C-Verletzungen stellen hoch instabile Situationen dar, die in aller Regel im Rahmen von Polytraumatisierungen auftreten und fast immer einer Osteosynthese bedürfen.

Der Begriff der Komplexverletzung beschreibt im Hinblick auf das Becken eine zusätzlich zur eigentlichen Beckenverletzung vorliegende Verletzung der intra- und parapelvinen Organe (bspw. Urethrarisse, Rektumverletzungen, Verletzungen der genitalen Weichteile, Blasenrupturen etc.). Diese Begrifflichkeit ist abzugrenzen von denen der Polytraumatisierung oder der Mehrfachverletzung, da sie sich rein auf die pelvinen Begleitverletzungen begrenzt.

\section{Azetabulum}

Auch hier sollte zunächst eine Beckenübersicht angefertigt werden (ggf. bereits mit Referenzkugel zur Planung einer Hüfttotalendoprothese, falls eine Schenkelhalsfraktur vorliegen sollte). Zusätzlich sind zur besseren Beurteilung Ala- und Obturatoraufnahmen indiziert. Hierbei handelt es sich um Schrägaufnahmen, bei denen das Foramen obturatum (Obturatoraufnahme) bzw. das Ala ossis ilii orthogonal eingestellt wird (siehe $\bullet$ Abb. 3 ).

Dadurch lassen sich Frakturen von vorderer und hinterer Wand besser gegen die Frakturen des vorderen oder hinteren Pfeilers abgrenzen bzw. überhaupt erst erkennen. Grundsätzlich lassen diese Projektionseinstellungen auch eine Beurteilung der Gelenkfläche hinsichtlich einer Stufen- oder Spaltbildung zu.

Aufgrund der Komplexität des Gelenkes, den vielen überlagernden Strukturen und der Notwendigkeit einer differenzierten Darstellung der Frakturmorphologie für die Operationsplanung ist es mittlerweile als Standard anzusehen, bei Azetabulumfrakturen eine CT-Diagnostik durchzuführen.

\section{Klassifikation}

Die bis heute gängige Klassifikation der Frakturen des Azetabulums wurde bereits 1964 durch Letournel und Judet beschrieben [4]. Grundlage dieser Klassifikation ist die biomechanische Unterscheidung eines vorderen von einem hinteren Pfeiler des Gelenkes. Über die Einheit dieser beiden Pfeiler erfolgt die Kraftübertragung von der unteren Extremität auf den hinteren Beckenring. Nicht lasttragende Anteile werden als vordere bzw. hintere Wand erfasst.

Die Klassifikation nach Judet unterscheidet 5 Grundtypen: vordere und hintere Wand oder Pfeiler und Querfrakturen. Zudem sind 5 sog. assoziierte Frakturen definiert, bei denen Frakturkombinationen oder schräge Frakturverläufe vorliegen. Eine Übersicht über die Frak- 

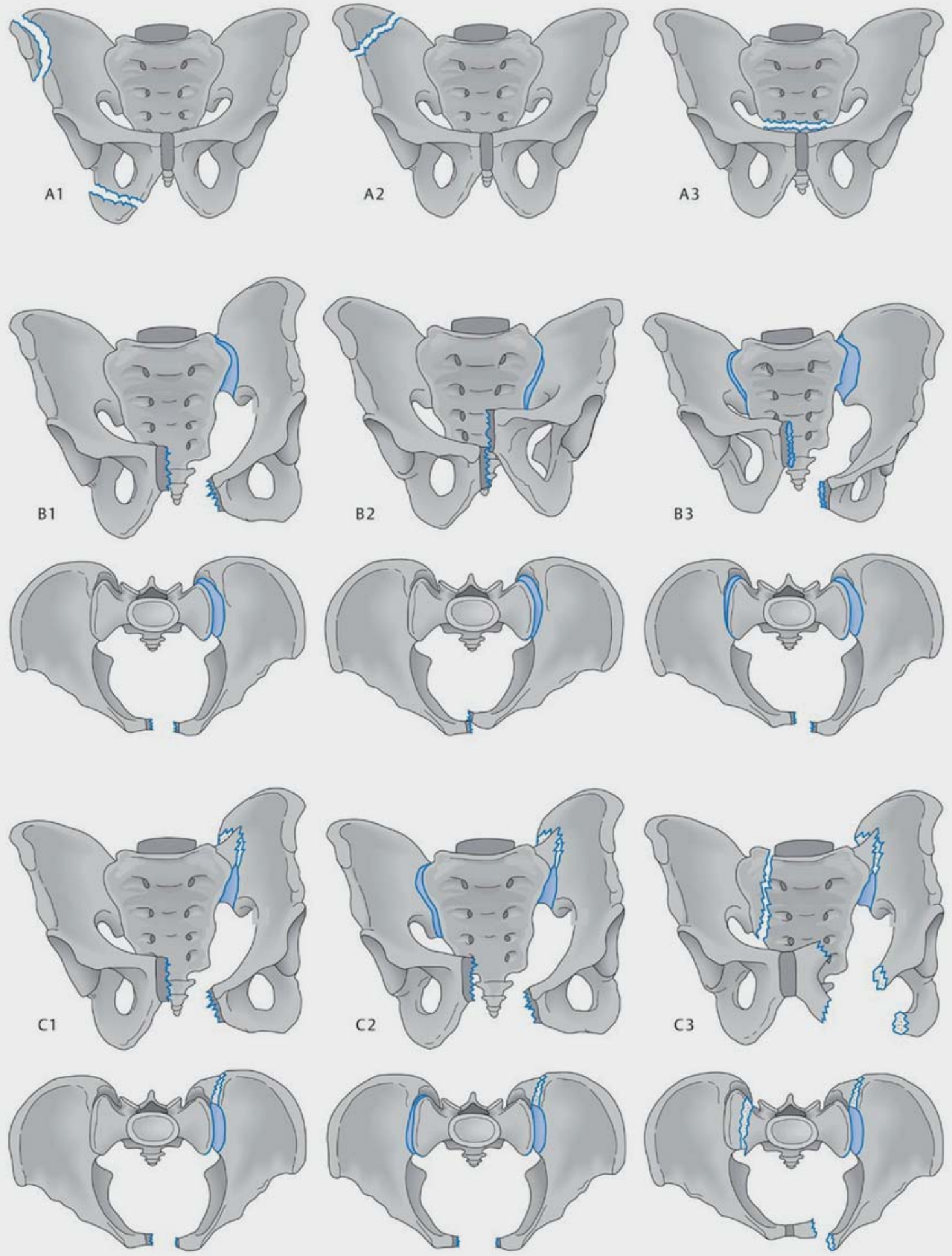

- Abb. 2 AO-Klassifikation der Beckenringverletzungen (aus: Wirbel R, Pohlemann T. Klassifikation der Beckenringverletzungen; Praxis der Orthopädie und Unfallchirurgie. 3. Aufl. Stuttgart: Thieme; 2013). 

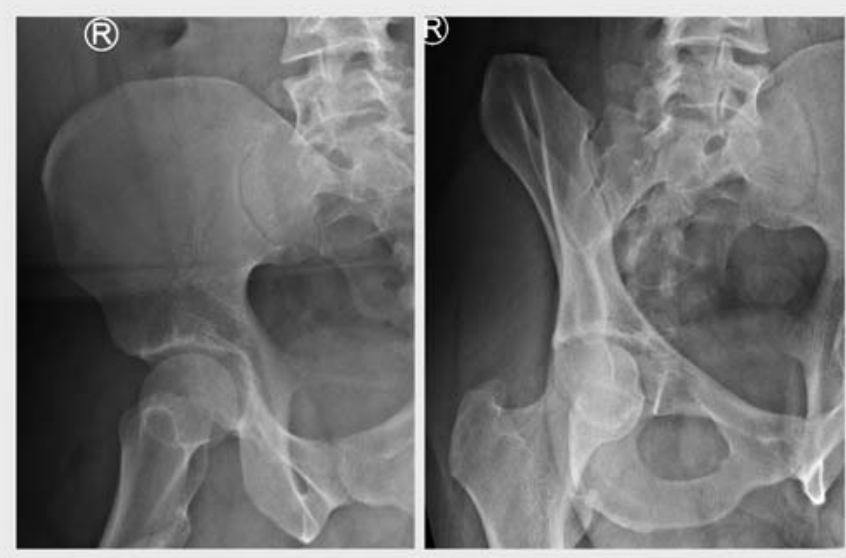

- Abb. 3 a Alaprojektion, b Obturatorprojektion.

turtypen gibt $\mathbf{A}$ Abb. 4. Die Durchführung der Klassifikation ist aufgrund der komplizierten Anatomie und der komplexen Frakturtypen anspruchsvoll und mit einer erheblichen Interobserver-Variabilität behaftet [5]. Ein Vorschlag für eine strukturierte Klassifikation der Frakturen zur Standardisierung der Befundung anhand von definierten CT-Schnitten wurde 2013 veröffentlicht [6].

In Verwendung ist auch eine AO-Klassifikation, die im Wesentlichen die Einteilung nach Letournel und Judet übernimmt und in das bekannte Schema systematisiert.

\section{Computertomografie}

In der CT lässt sich die knöcherne Anatomie sowie der Frakturverlauf exakt darstellen und damit zum einen ein sicherer Frakturnachweis führen und zum anderen eine korrekte Klassifizierung durchführen. Ebenso ist eine Beurteilung der Stellung der Sakroiliakalgelenke (SI-Gelenke) im Seitenvergleich möglich, genauso wie eine Analyse und Messung des Symphysenspaltes.

Insuffizienzfrakturen im frühen Stadium lassen sich projektionsradiografisch nicht suffizient beurteilen bzw. erkennen. Bei persistierendem Frakturverdacht trotz unauffälligen Röntgens sollte hier frühzeitig eine CT- oder (besser) MRT-Diagnostik erfolgen. Die MRT hat gegenüber der CT den Vorteil des Nachweises des intraossären Hämatoms als Zeichen der pathologischen Fraktur. Gegebenenfalls kann nämlich auch mit der CT ein Frakturnachweis nicht gelingen, wenn die Gefügestörung des Knochens noch nicht zu einer Dislokation des Knochens geführt haben sollte. Eine zusätzliche und im Vergleich zur MRT besser verfügbare Option könnte in Zukunft die Dual-Energy-CT darstellen, in der analog zur MRT das Frakturödem im Bereich des Sakrums visualisiert wird [7].

Bei hämodynamisch instabilen Patienten im Rahmen einer Polytraumatisierung steht selbstverständlich die
Notfalldiagnostik mit kontrastmittelverstärkter Ganzkörper-CT im Vordergrund. Bei diesen Patienten, die über den Schockraum aufgenommen werden und bei denen eine externe Beckenkompression (Beckengurt) angelegt wurde, stellt sich die Frage nach dem korrekten Vorgehen hinsichtlich der Diagnostik. Hintergrund ist die Möglichkeit, dass eine Open-Book-Verletzung ohne knöcherne Verletzung durch einen korrekt angelegten Beckengurt reponiert wird und damit im CT mit angelegtem Gurt nicht dargestellt werden kann. Entsprechende algorithmische Herangehensweisen wurden beschrieben [8]. Im hauseigenen Vorgehen hat sich daher die in $\mathbf{A b b} \mathbf{5}$ skizzierte Vorgehensweise etabliert. Hiermit soll sowohl sichergestellt werden, dass eine instabile Beckenverletzung möglichst frühzeitig und effizient komprimiert wird, als auch, dass eine vollständige Diagnostik durchgeführt werden kann.

\section{Intraoperative Bildgebung}

\section{Beckenring}

Um bei der operativen Versorgung von Beckenringfrakturen die Möglichkeit einer suffizienten Projektion mit Durchführung der Inlet- und Outlet-Aufnahmen zu ermöglichen, ist es notwendig, den Patienten so weit wie möglich an das fußseitige Ende des Tisches zu lagern. Hierzu sind spezielle - idealerweise mit Carbonliegefläche - Tische notwendig, die nach distal verlängert werden können. Vor dem Abdecken sollte sichergestellt werden, dass der verwendete C-Bogen weit genug eingeschwenkt werden kann, um diese Projektionen einzustellen. Je nach individueller Anatomie ist das notwendige Ausmaß des Einschwenkens unterschiedlich, beträgt aber i.d.R. zwischen 35 und $45^{\circ}$ nach kranial und kaudal (siehe Abb. 6a-c, 7a, b und 8a,b).

\section{Merke}

Qualitätskriterien für diese Einstellungen sind für die Outlet-Projektion die orthograde Darstellung der Beckeneingangsebene mit Überlagerung der kranialen und kaudalen Anteile des vorderen Beckenringes und senkrechter Darstellung der Vorderkante S I. In der Inlet-Projektion stellen sich die Foramina obturatoria beidseitig maximal in kraniokaudaler Ausdehnung dar, die Symphyse ist senkrecht getroffen.

Bei Osteosynthesen des vorderen Beckenringes - häufig die Osteosynthese der Symphyse oder des Schambeines - ist i. d. R. die intraoperative Röntgenkontrolle unkompliziert und mit Inlet- und Outlet-Aufnahmen problemlos abzubilden. Hiermit ist eine suffiziente und vollständige Beurteilung der Reposition und Implantatlage möglich.

Komplexer ist die Situation am hinteren Beckenring, insbesondere bei der perkutanen Positionierung von Schrauben durch das SI-Gelenk. Hier ist die exakte seitliche so- 




- Abb. 4 Klassifikation der Azetabulumfrakturen nach Letournel (aus: Stannard JP, Schmidt AH. Surgical Treatment of Orthopaedic Trauma. Stuttgart: Thieme; 2007). 


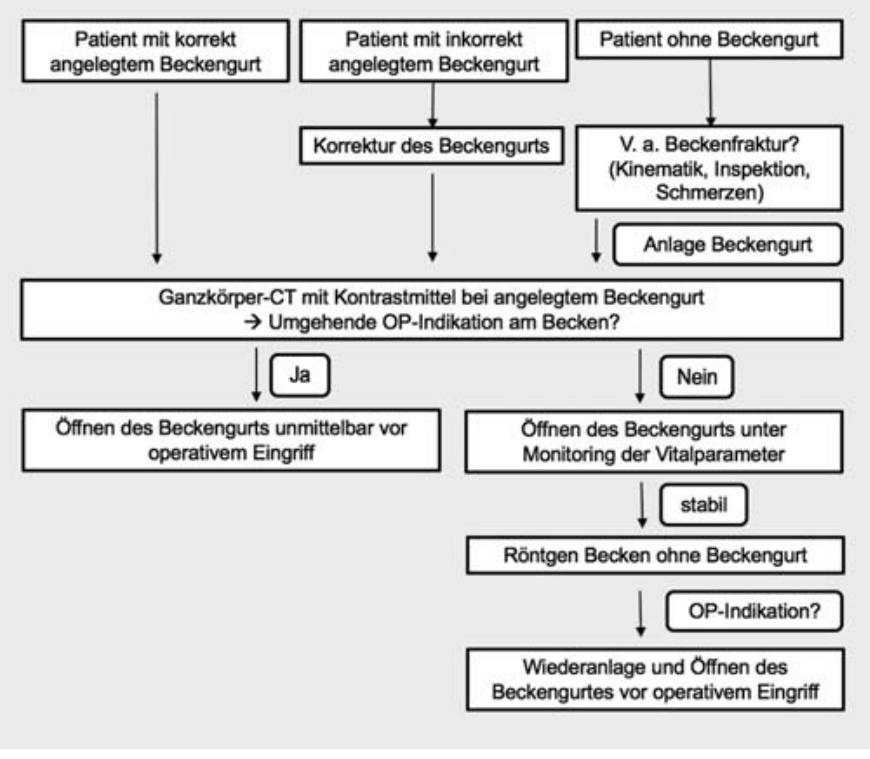

- Abb. 5 Algorithmus zur radiologischen Diagnostik bei anliegendem Beckengurt.

wie axiale Einstellung des Sakrums notwendig, um eine intraspinale bzw. intraforaminale Lage der Schrauben auszuschließen (siehe $>$ Abb. 9).

Die axiale Einstellung entspricht i.d. R. der Outlet-Projektion, kann jedoch, je nach individueller Anatomie, auch von dieser abweichen. Hier ist besonders die Angulation der Deckplatte von S I von Bedeutung. Hier kann es vorkommen, dass eine axiale Einstellung nicht möglich ist, da der C-Bogen zuvor am Torso anstößt. Überlagerungen durch Darmgase, schlechte Knochenqualität und Dysmorphien des Sakrums mit Irregularitäten der SI-Gelenke sind weitere Faktoren, welche die intraoperative Darstellungsqualität einschränken können. Auch eine Adipositas mit entsprechender Weichteilüberlagerung kann die Be- urteilung sehr stark beeinträchtigen. Qualitätskriterien für diese Einstellung sind, dass sowohl die Alae auf beiden Seiten des Sakrums als auch die Hüftköpfe aufeinander projiziert werden. Zudem sollte sowohl die Deckplatte S I orthograd getroffen als auch die kraniale Begrenzung der Alae eindeutig sichtbar sein. Dies muss beim Einbringen der Schrauben oder von deren Führungsdrähten unbedingt beachtet werden, da hier die Nervenwurzeln L IV und $\mathrm{L} V$ direkt dem Knochen anliegend verlaufen und durch die Implantate verletzt werden können (siehe - Abb. 10).

\section{Merke}

Für die konventionelle Positionierung von SI-Schrauben muss die seitliche Ebene exakt eingestellt werden können!

Eine entsprechende präoperative Analyse der Bildgebung mit entsprechender Planung und Vorbereitung des Patienten ist essenziell, um die Positionierung erfolgreich durchführen zu können.

\section{Azetabulum}

Die Lagerung von Patienten, die einen operativen Eingriff am Azetabulum erhalten, ist ebenfalls vorher zu planen. Zu bedenken ist der vorgesehene operative Zugang in Abhängigkeit von der durch die Operation zu adressierenden Struktur, vor allem, ob der vordere oder hintere Pfeiler operiert werden soll. Der vordere Pfeiler wird i.d.R. über einen Zugang in Rückenlage erreicht (z.B. Stoppa-Zugang), der hintere Pfeiler über einen dorsalen Zugang (z. B. nach Kocher-Langenbeck) in Seitenlage.

Auch hier hat sich die Verwendung eines Carbontisches bewährt, bei dem es möglich ist, die betreffende Seite in Ala- und Obturatoreinstellung zu durchleuchten. Andernfalls sollte ebenfalls vor dem Abdecken überprüft werden, dass keine röntgendichten Anbauteile des Tisches im Strahlengang der geplanten Projektionen liegen. Für
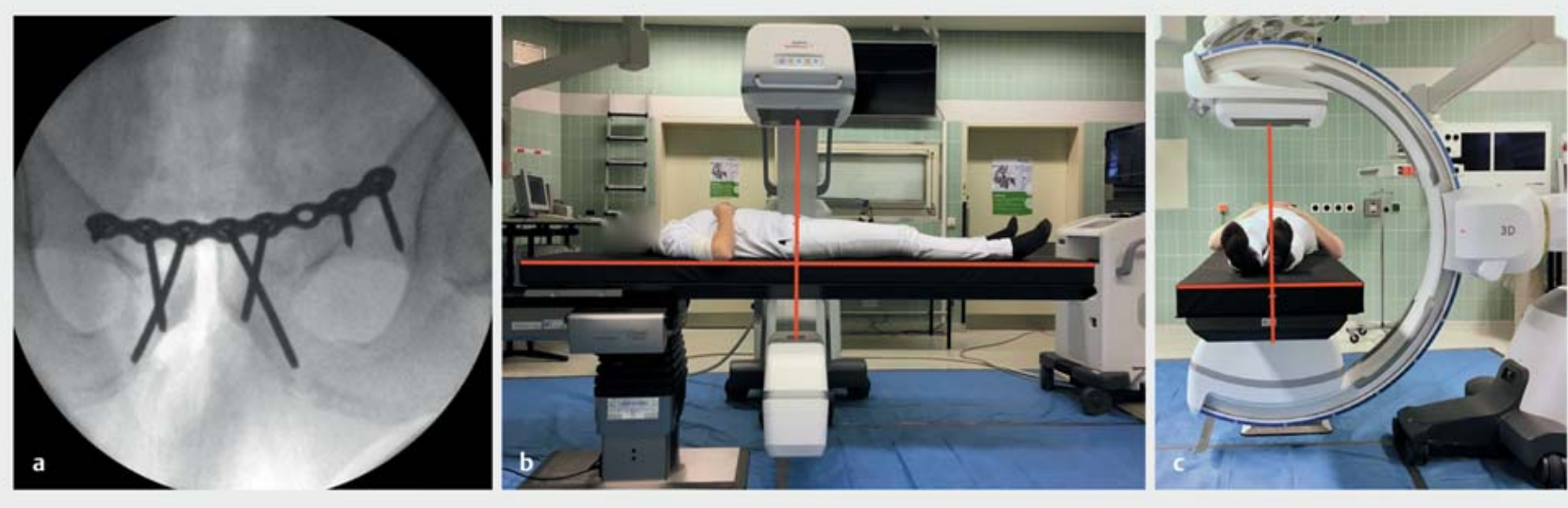

Abb. 6 a-c Intraoperative a.-p. Projektion mit der entsprechenden Einstellung des C-Bogens. 

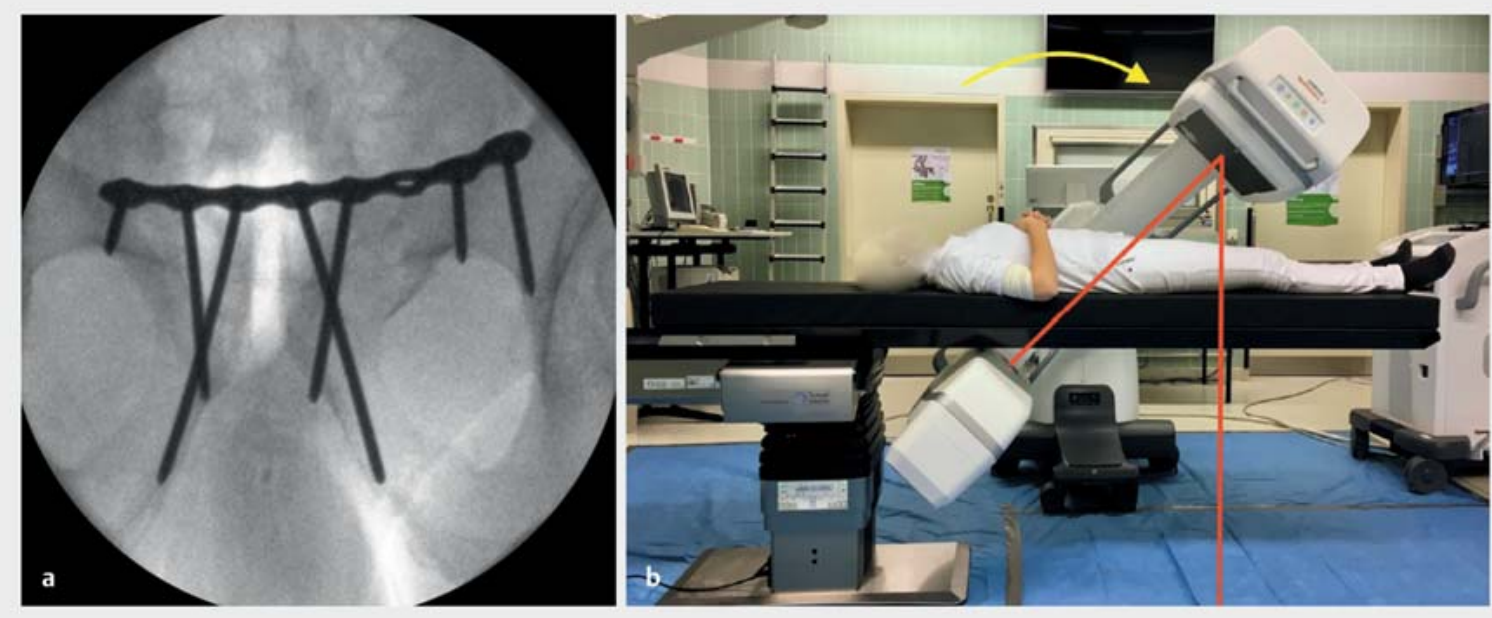

- Abb. 7 a,b Intraoperative Inlet-Projektion mit der entsprechenden Einstellung des C-Bogens.
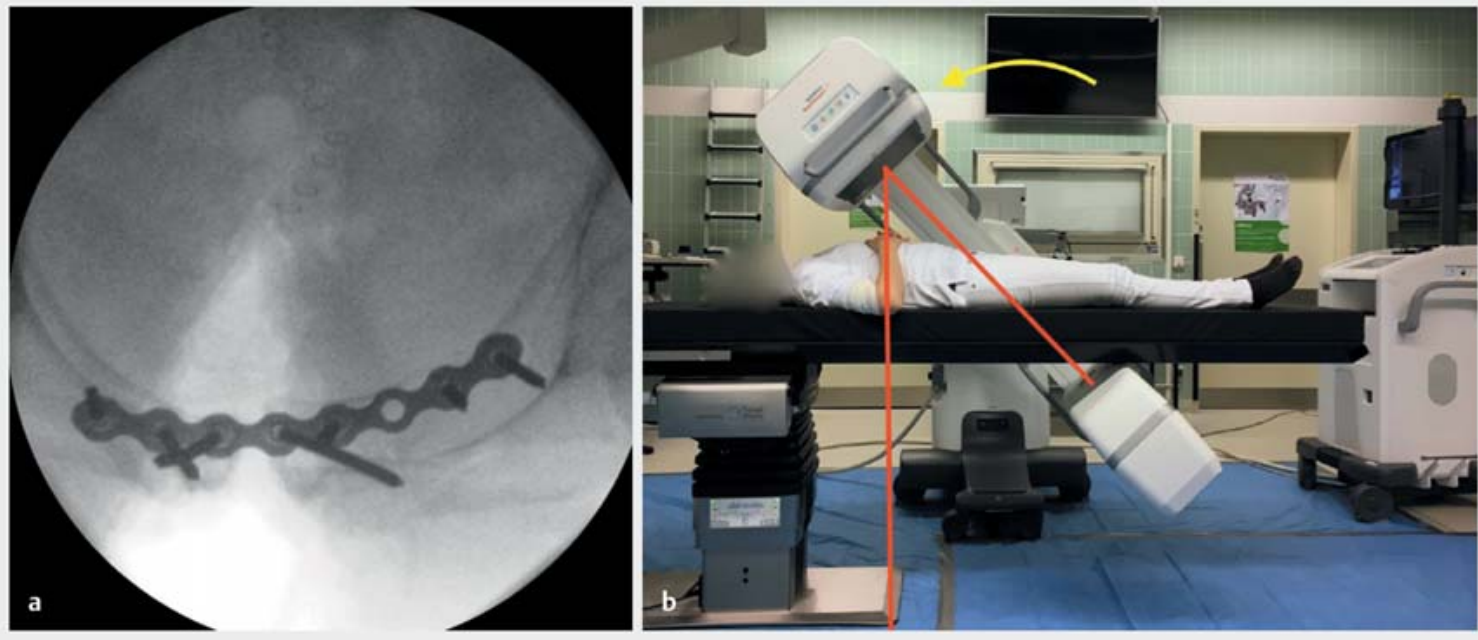

- Abb. 8 a,b Intraoperative Outlet-Projektion mit der entsprechenden Einstellung des C-Bogens.

die Projektionen sind i.d.R. Orbitaleinstellungen von $\pm 35-45^{\circ}$ notwendig (siehe $>$ Abb. 10 und 11). Bei Seitenlagerung ist für die korrekte Einstellung der Projektionen eine atypische Positionierung des C-Bogens notwendig, diese sollte auf jeden Fall erreicht werden können.

\section{Merke}

Qualitätskriterien der Schrägeinstellungen sind die maximale Ausdehnung des Iliums in der Alaprojektion mit Überlagerung der lateralen und medialen Anteile des Foramen obturatum. Für die Obturatoreinstellung ist das Foramen obturatum maximal ausgedehnt, während das Os ilium tangential getroffen wird.
Eine atypische Einstellung stellt die sog. Punktprojektion dar. Bei Schraubenverläufen nahe am Gelenkspalt kann es in den Standardprojektionen manchmal schwierig sein, einen intraartikulären Schraubenverlauf auszuschließen. In der Regel ist es aber möglich, die Schraube möglichst senkrecht einzustellen, sodass sich diese nur noch als Punkt projiziert (siehe A Abb. 12). Damit kann der extraartikuläre Verlauf sichergestellt werden.

Merke

Die Lage einzelner Schrauben kann durch atypische Projektionen verifiziert werden, wenn eine entsprechende Einstellung möglich ist! 

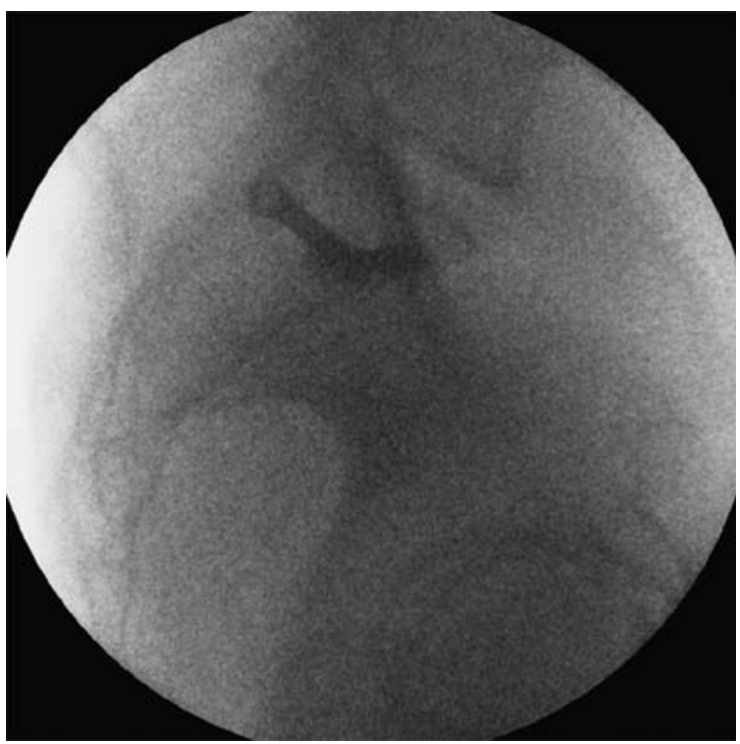

- Abb. 9 Seitliche Einstellung des Os sacrum mit einliegenden SI-Schrauben beidseitig.

\section{Intraoperative 3-D-Bildgebung und Navigation}

Die intraoperative 3-D-Bildgebung ist bei Rekonstruktionen komplexer Gelenkfrakturen ein zusätzliches und wertvolles Hilfsmittel, um die Reposition und Implantatlage überprüfen zu können $[9,10]$. Die anatomische Rekonstruktion von Gelenkflächen und die sicher extraartikuläre Lage von Implantaten sind die relevanten Kriterien, um den Erfolg einer Osteosynthese zu sichern. In Abhängigkeit von der anatomischen Region und Situation können diese Parameter nicht immer vollständig in den 2-D-Projektionen beurteilt werden. Traditionell werden in Situationen, die intraoperativ nicht sicher zu beur- teilen sind, postoperative CT-Kontrollen durchgeführt. Bei signifikanten Implantatfehllagen oder verbliebenen Gelenkstufen oder -spalten ist dann ein Revisionseingriff mit erneuter Narkose notwendig. Die intraoperative 3-D-Kontrolle ermöglicht die umgehende Sicherung der Reposition und Implantatlage, sodass ggf. umgehend eine Revision erfolgen kann.

Im eigenen Vorgehen erfolgt bei allen Osteosynthesen am Azetabulum eine intraoperative 3-D-Kontrolle, wenn der Operateur in den 2-D-Projektionen die Reposition und Implantatlage als suffizient bewertet. In der Langzeitbeobachtung am eigenen Patientengut erfolgten hierdurch am Azetabulum in über 15\% der Fälle umgehende Verbesserungen der Reposition oder der Implantatlage, wodurch Revisionsoperationen vermieden werden konnten [11].

\section{Merke \\ Mit der intraoperativen 3-D-Bildgebung können Revisionsoperationen vermieden werden!}

Bei operativen Maßnahmen am hinteren Beckenring, insbesondere bei der perkutanen Schraubenpositionierung in den SI-Gelenken, kann die intraoperative 3-D-Bildgebung ebenfalls hilfreiche Zusatzinformationen liefern. Damit ist der extraforaminale Verlauf der Schrauben ebenso sicherzustellen, wie die extraspinale Lage [12].

Zusätzlich kann ein intraoperativ gewonnener 3-D-Datensatz als Basis für die strahlungsfreie Navigation verwendet werden (siehe $>$ Abb. 13). Hierfür wird nach Lagerung des Patienten eine Referenzbasis in der Crista iliaca über eine Schanz-Schraube befestigt und ein 3-D-Scan des betreffenden anatomischen Bereiches durchgeführt. Durch am C-Bogen befestigte Referenzmarker wird durch

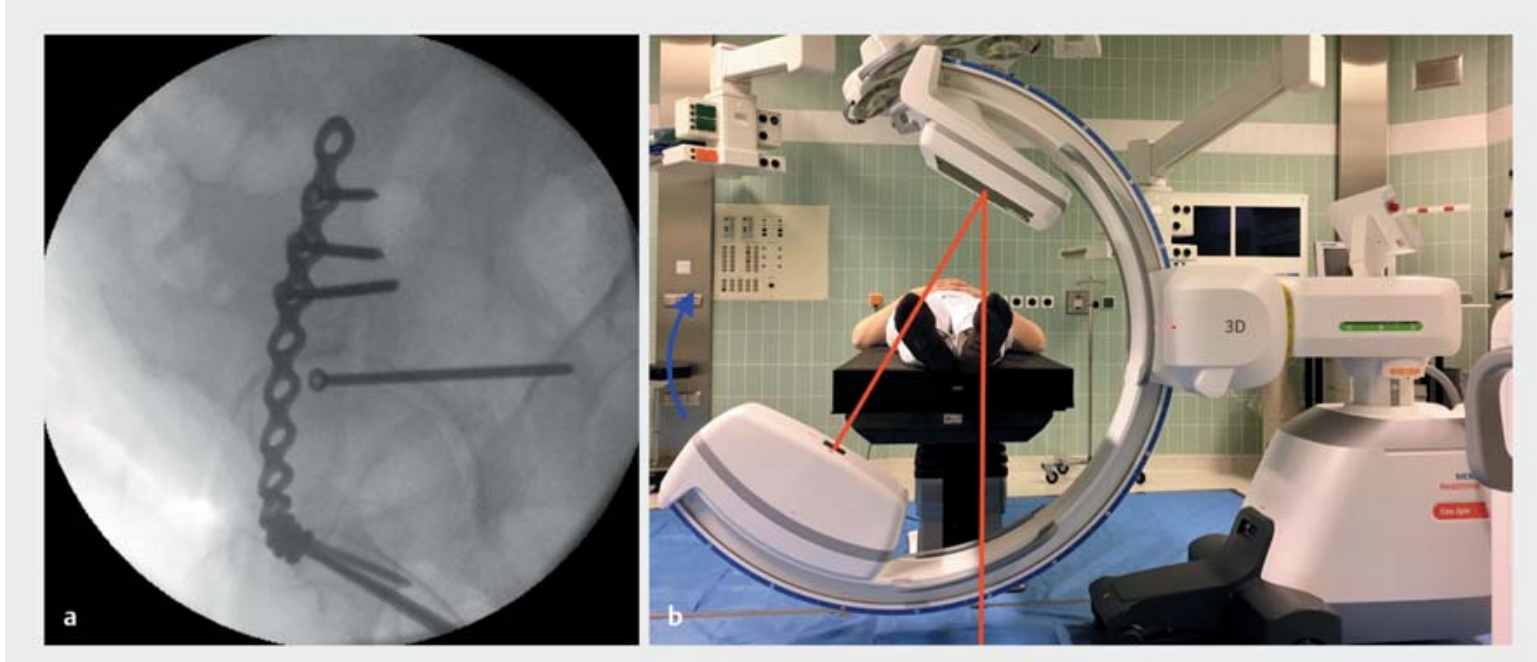

- Abb. 10 a,b Intraoperative Alaprojektion mit entsprechender Einstellung des C-Bogens. 


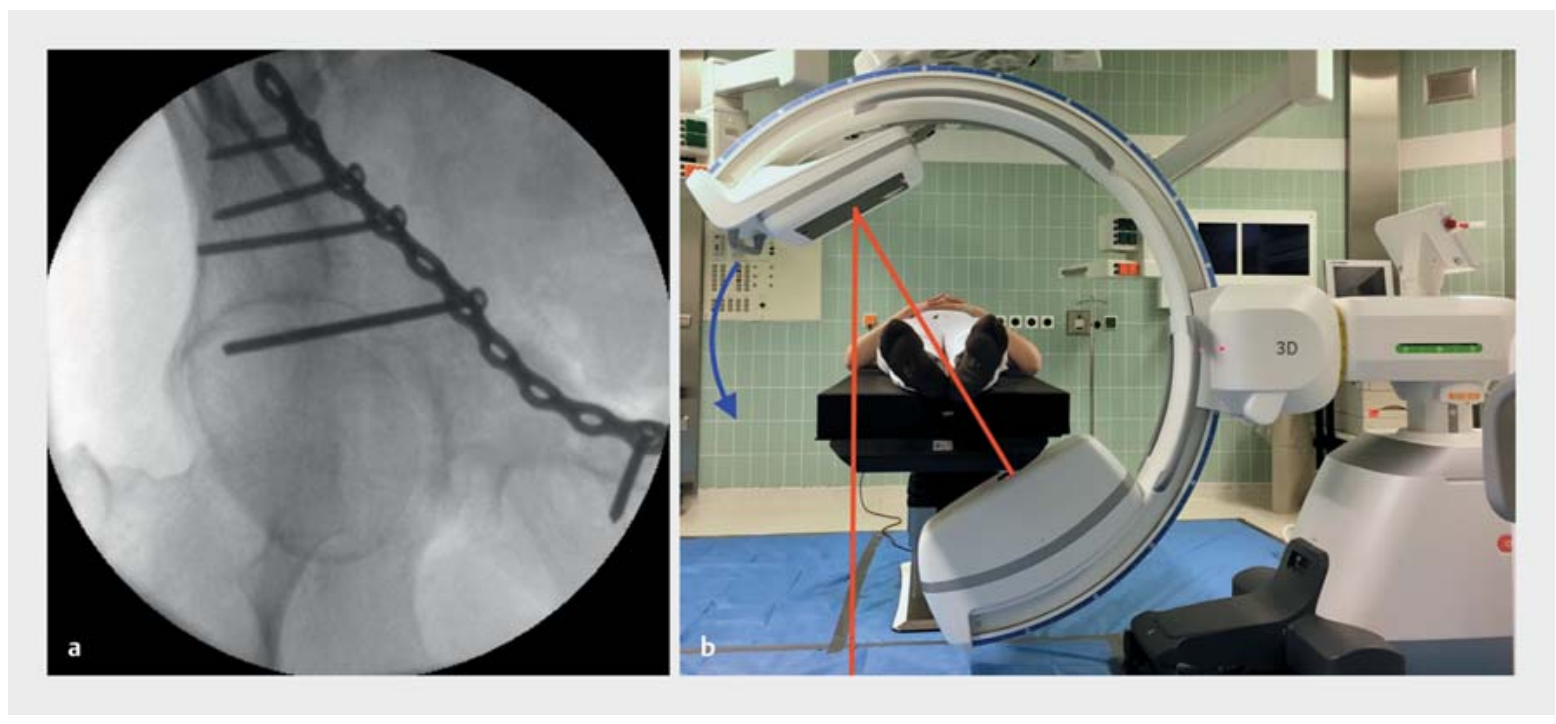

- Abb. 11 a,b Intraoperative Obturatorprojektion mit entsprechender Einstellung des C-Bogens.

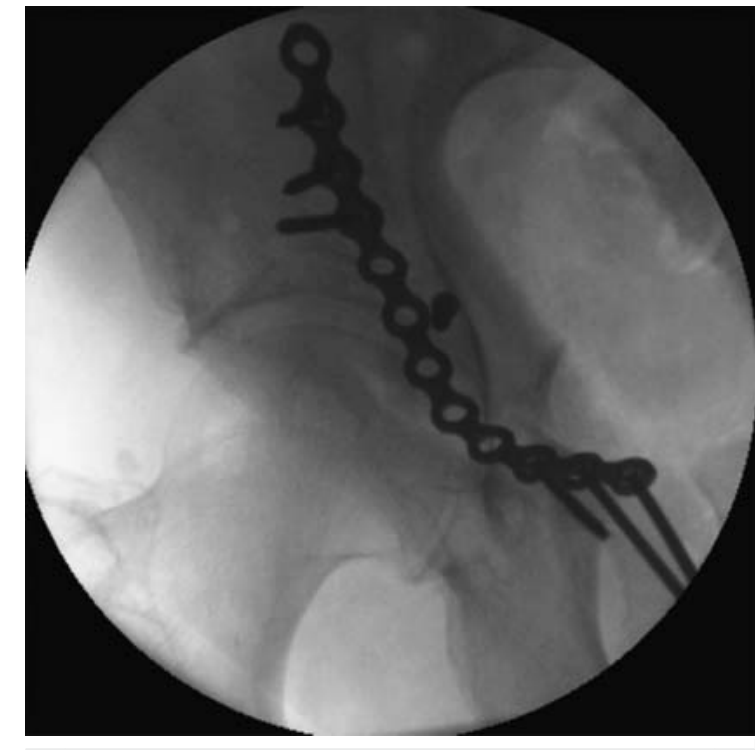

Abb. 12 Atypische Einstellung des Azetabulums. Der Zentralstrahl verläuft parallel zur Schraube.

eine Stereoinfrarotkamera eine Referenzierung durchgeführt, sodass im Anschluss mit entsprechenden Instrumenten eine Livenavigation im Bilddatensatz erfolgen kann. Solange sich die Anatomie nicht ändert, ist über diese Lösung eine konstante Anzeige von Instrumentenund Implantatposition im Datensatz möglich. Dadurch lässt sich die Positionierung der Implantate insbesondere bei komplexer Anatomie (z. B. bei Sakrumdysmorphie) erheblich vereinfachen.

Ist die Verwendung eines 3-D-C-Bogens geplant, sollte ebenfalls entsprechend vor dem Abdecken geprüft wer- den, ob die Orbitalbewegung problemlos erfolgen kann. Auch bei der Verwendung der intraoperativen 3-D-Bildgebung mit einem mobilen C-Bogen sollte man sich über mögliche Einschränkungen bewusst sein. So ist durch die begrenzte Größe des abbildbaren Volumens, das i.d.R. einem Würfel mit einer Kantenlänge von 12-14 cm entspricht, nur die Abbildung eines SI-Gelenkes oder Azetabulums möglich. Zudem wird die Beurteilbarkeit der Aufnahmen durch das Vorhandensein von Artefakten bei Implantaten im Strahlengang die Bildqualität erheblich eingeschränkt.

Für spezialisierte Zentren ist aktuell als technisch fortschrittlichste Variante der intraoperativen Schnittbildgebung die Verwendung eines intraoperativen CTs zur Navigation und Kontrolle von Reposition und Implantatlage möglich. Hierbei handelt es sich i.d.R. um speziell für den intraoperativen Einsatz konzipierte Geräte, die sich gut in das Setting integrieren lassen (z. B. das Gerät „Airo Mobile intraoperative CT“ der Firma Brainlab; siehe Abb. 14 und 15).

\section{Postoperative Bildgebung}

Das Ausmaß der notwendigen postoperativen Bildgebung hängt zum einen von der durchgeführten operativen Maßnahme, zum anderen von der intraoperativ erfolgten Diagnostik ab. Zusammenfassend lässt sich sagen, dass mit der postoperativen Kontrolle eine Sicherstellung des Operationsergebnisses erfolgen sollte. Üblicherweise wird dies nach Mobilisierung mit dem erlaubten Belastungs- und Bewegungsumfang durchgeführt und damit neben der Reposition und Implantatlage die Stabilität des Konstruktes geprüft. 

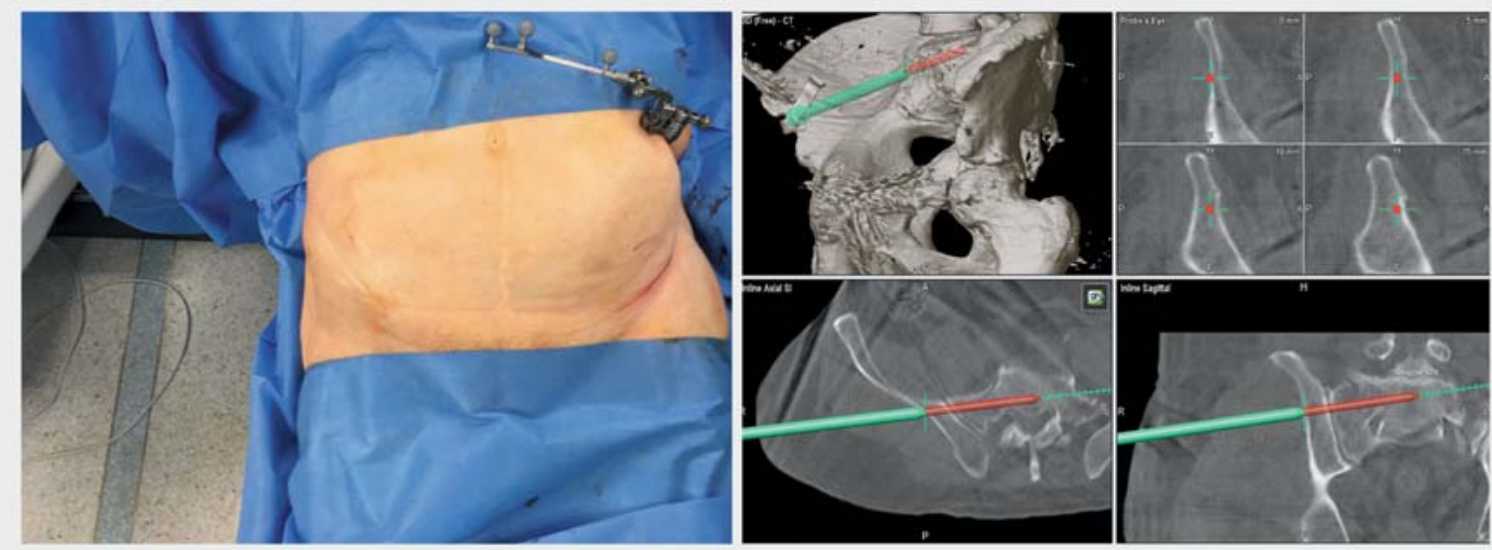

Abb. 13 Operationssitus mit befestigter Referenz für die Navigation sowie Ansicht der Navigation in einem intraoperativ gewonnenen 3-D-Datensatz.

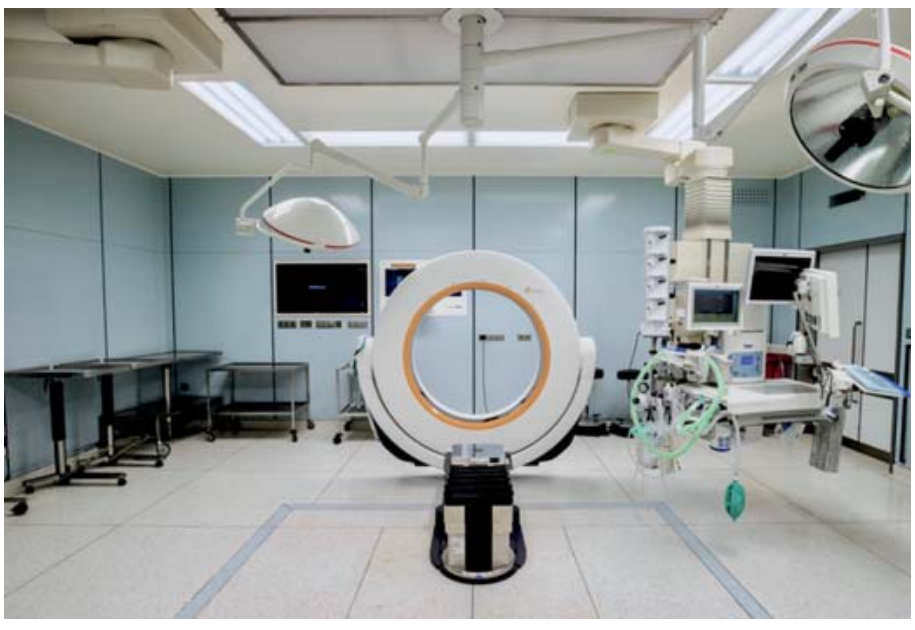

- Abb. 14 Mobiles intraoperatives CT „Brainlab Airo“.

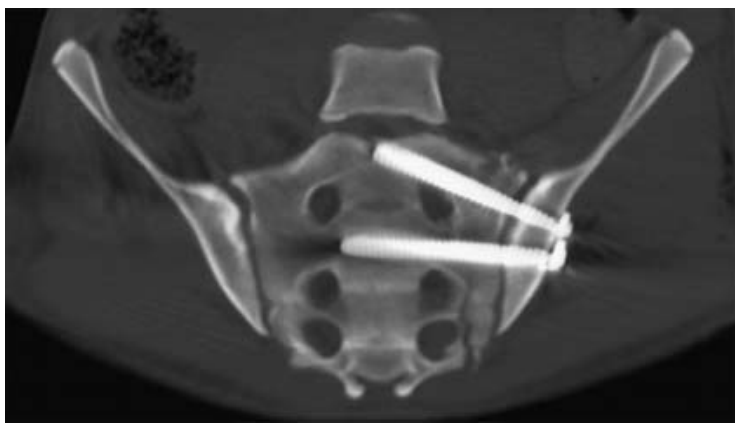

- Abb. 15 Intraoperative CT-Kontrolle der Implantatlage bei Sakrumdysmorphie.
Im hauseigenen Vorgehen erfolgt die Röntgenkontrolle nach Drainagenzug und - sofern möglich - Mobilisierung.

Bei Azetabulumfrakturen erfolgt analog der präoperativen Diagnostik die Kontrolle in a.-p. Projektion sowie in Ala- und Obturatoraufnahme. Wurde intraoperativ kein 3-D-Scan in guter Qualität durchgeführt, erfolgt postoperativ eine CT-Aufnahme zur sicheren Kontrolle der Reposition.

Bei Beckenringfrakturen erfolgen neben a.-p. Projektion Inlet- und Outlet-Aufnahmen. Wurde bei SI-Schraubenpositionierung kein 3-D-Scan durchgeführt, erfolgt auch hier die postoperative CT-Kontrolle.

Eine besondere Möglichkeit der Röntgendiagnostik sind die sog. Belastungsaufnahmen bei Verdacht auf Instabilität einer Symphyenverletzung. Hier werden a.-p. Aufnahmen in Einbeinstand links und rechts durchgeführt (siehe $>$ Abb. 16). Dies eröffnet eine diagnostische Facette, die mit CT-Aufnahmen durch die notwendige Rückenlage nicht möglich ist.

Zur Verlaufskontrolle erfolgen i.d.R. 6 Wochen, 3 und 6 Monate postoperativ Röntgenaufnahmen in den beschriebenen Projektionen. Zeigen sich hier Auffälligkeiten oder gibt der Patient persistierende oder neu aufgetretene Beschwerden an, erfolgt eine CT-Diagnostik.

\section{Fazit}

In der präoperativen Diagnostik stehen neben der a.-p. „Standardbeckenübersicht“ verschiedene Schrägaufnahmen zur gezielten Abbildung der relevanten Strukturen zur Verfügung. Die Indikation zur CT sollte insbesondere bei Azetabulumfrakturen großzügig gestellt werden. 


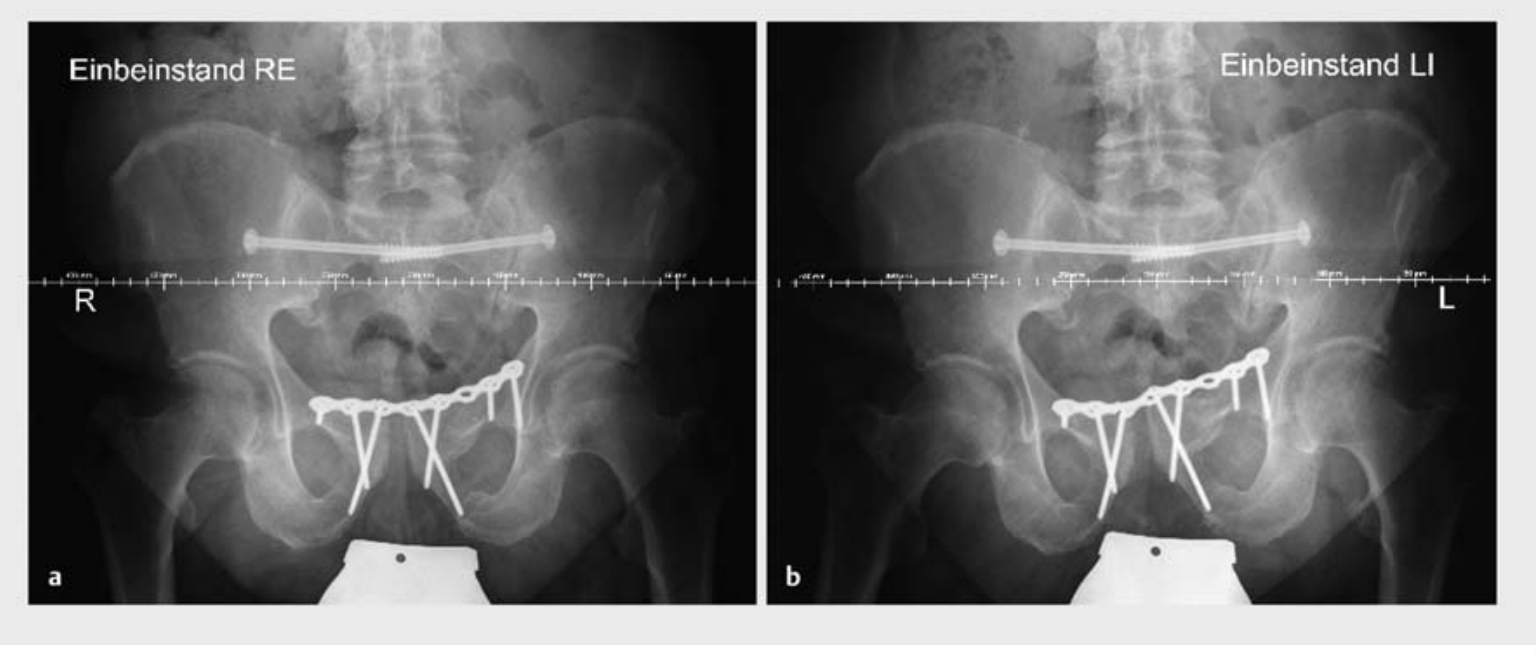

- Abb. 16 a,b Nachweis einer Symphyseninstabilität durch Röntgenaufnahmen im Einbeinstand.

Für die intraoperative Bildgebung sollte auf die korrekte Lagerung auf einem geeigneten OP-Tisch geachtet werden. Bei Osteosynthesen des Azetabulums sollte eine intraoperative 3-D-Bildgebung oder eine postoperative CT erfolgen. Zur Instrumentierung von SI-Schrauben ist, insbesondere bei anatomischen Varianten, die 3-D-gestützte Navigation ein gutes Hilfsmittel, um die Positionierung zu vereinfachen. Die radiologischen Verlaufskontrollen sollten sich bei unproblematischem Verlauf auf Röntgenaufnahmen in den jeweiligen Standardebenen beschränken. Bei Auffälligkeiten ist jedoch frühzeitig an die Durchführung einer CT zu denken.

\section{Interessenkonflikt}

Die Autoren erklären, dass sie in den vergangenen 3 Jahren Vortragshonorare und Forschungsunterstützung der Siemens Healthcare AG und Brainlab AG erhalten haben.

\section{Autorinnen/Autoren}
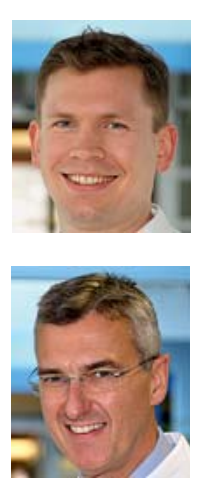

\section{Holger Keil}

Dr., Klinik für Unfallchirurgie und Orthopädie, BG Klinik Ludwigshafen

\section{Paul A. Grützner}

Prof. Dr., Klinik für Unfallchirurgie und Orthopädie, BG Klinik Ludwigshafen

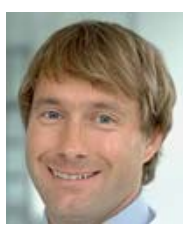

\section{Jochen Franke}

Dr., Klinik für Unfallchirurgie und Orthopädie, BG Klinik Ludwigshafen

\section{Korrespondenzadresse}

\section{Dr. Jochen Franke}

Klinik für Unfallchirurgie und Orthopädie

BG Klinik Ludwigshafen

Ludwig-Guttmann-Str. 13

67071 Ludwigshafen

jochen.franke@bgu-ludwigshafen.de

\section{Literatur}

[1] Tscherne H, Pohlemann T. Tscherne Unfallchirurgie. Heidelberg: Springer; 1997

[2] Shlamovitz GZ, Mower WR, Bergman J et al. How (un)useful is the pelvic ring stability examination in diagnosing mechanically unstable pelvic fractures in blunt trauma patients? J Trauma 2009; 66: 815-820

[3] Wohlrath B, Trentzsch H, Hoffmann R et al. Präklinische und klinische Versorgung der instabilen Beckenverletzung. Unfallchirurg 2016; 119: 755-762

[4] Judet R, Judet J, Letournel E. Fractures of the acetabulum: classification and surgical approaches for open reduction. Preliminary report. J Bone Joint Surg Am 1964; 46: 1615-1646

[5] Visutipol B, Chobtangsin P, Ketmalasiri B et al. Evaluation of Letournel and Judet classification of acetabular fracture with plain radiographs and three-dimensional computerized tomographic scan. J Orthop Surg (Hong Kong) 2000; 8: 33-37

[6] Schäffler A, Fensky F, Knöschke D et al. CT-basierte Klassifikationshilfe für Acetabulumfrakturen: Evaluation und klinische Erprobung. Unfallchirurg 2013; 116: 1006-1014 
[7] Hackenbroch C, Riesner H-], Lang P et al. Dual Energy CT - a novel technique for diagnostic testing of fragility fractures of the pelvis. Z Orthop Unfall 2017; 155: 27-34

[8] Schweigkofler U, Wohlrath B, Paffrath T et al. „Clear-the-Pelvis-Algorithmus": Handlungsempfehlung zur Freigabe des Beckens nach nicht invasiver Stabilisierung mittels Beckengurt im Rahmen der Schockraumversorgung. Z Orthop Unfall 2016; 154: 470-476

[9] Beerekamp MSH, Ubbink DT, Maas M et al. Fracture surgery of the extremities with the intra-operative use of 3D-RX: a randomized multicenter trial (EF3X-trial). BMC Musculoskelet Disord 2011; 12: 151

[10] Beerekamp MS, Sulkers GS, Ubbink DT et al. Accuracy and consequences of 3D-fluoroscopy in upper and lower extremity fracture treatment: a systematic review. Eur J Radiol 2012; 81: 4019-4028
[11] von Recum J, Wendl K, Vock B et al. Die intraoperative 3D-CBogen-Anwendung. Unfallchirurg 2012; 115: 196-201

[12] Zwingmann J, Hauschild O, Bode G et al. Malposition and revision rates of different imaging modalities for percutaneous iliosacral screw fixation following pelvic fractures: a systematic review and meta-analysis. Arch Orthop Trauma Surg 2013; 133: $1257-1265$

\section{Bibliografie}

DOI https://doi.org/10.1055/s-0044-100157

OP-JOURNAL 2018; 34: 5-16 @ Georg Thieme Verlag KG Stuttgart · New York ISSN 0178-1715 